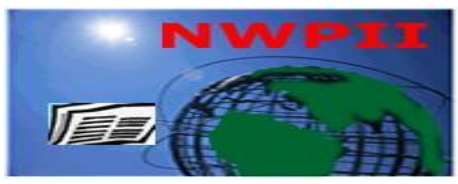

American Journal of Biomedical Sciences

ISSN: 1937-9080

nwpii.com/ajbms

\title{
Designing and Implementation of Liquid Electronic Circuits Using Implantable Material-First Step towards Human-circuit Interface
}

\section{Killol Pandya}

Department of Electronics and Communication, CHARUSAT University,Off-Nadiad Petlad road,Pin-388421, Gujarat, India

"Corresponding Author

Killol Pandya

Department of Electronics and Communication

CHARUSAT University

Off-Nadiad Petlad road, Pin-388421

Gujarat

India

Email: killolpandya.ec@charusat.ac.in

Phone:02697-265011/21

Received: 23 September 2017; | Revised: 02 November 2017; | Accepted: 22 December 2017;

\begin{abstract}
Biomedical engineering is the immerging domain which applies the concepts of engineering into biomedical science. A first step towards development of pure biological human implantable electronic circuit has been effectively proposed in this paper. A resistor transistor logic circuit using liquid medium (synthetic plasma) has been successfully developed, analyzed and presented in this paper. To justify the stability of the liquid, different density synthetic plasma has been analyzed with similar experiments. Once desired response from liquid circuit has been achieved, a novel biological resistor transistor logic integrated circuit has been proposed and analyzed. Presented work claims about implantation of similar kinds of circuits in to the human body with proper mechanical instrumentation and clinical care.
\end{abstract}

Keywords: Biomedical science, synthetic plasma, liquid electronic circuits, human body, liquid integrated circuit

\section{Introduction}

Shivprasad Kosta and his team (2011) presented a liquid memristor concept which claims that liquid based electronic circuits are feasible with proper instrumentation ${ }^{[1]}$. Shivprasad Kosta et al(2013) ${ }^{[2]}$ used human body parts like skin, live tissues to realize a memristor circuit. The authors (2012) have given the novel idea to develop human 
machine interface technology and cyborg implantation. S P Kosta et al successfully shown that human tissue, skin, live cell or human blood (artificial blood) could be an appropriate medium for electrical conductivity ${ }^{[3-5]}$. In 2005, Marc Simon Wegmueller et al. investigated that human body could be a transmission medium at low frequency for low power human LANs ${ }^{[6]}$. The human body is neutral in nature but every human being live cells are surrounded by a tissue made up of a fatty acid bilayer with proteins implanted in it. The tissue has a role as an insulator and a diffusion barrier to the movement of ions. The ions are pushed by the ions proteins across the tissue to get concentration gradients across the cell and ion channels allow ions to move across the tissue/cell down those concentration gradients. Ion particles and ion channels are electrically same to a set of batteries and resistors in the tissue. ${ }^{[6-7]}$. Yogesh Patil and his team (2013) stated that the nano particles are always present in to the human body. Further they said that nano particles are very much smaller in size so that they can easily travel through any human blood cell and may cause damage. So in order to reduce its effect, successful absorption of metal into the human body is a very effective technique. In short they also believe that human body is a very good conductor $^{[8]}$. In the past, a few researchers (2015) have done noticeable experiment to charge low voltage battery automatically using human body surface (skin). The authors confessed that sensors could be automatically rechargeable because there is no alternative way to recharge or change the battery on the sensor nodes. But keeping dynamic coordinator, it is very convenient to cope up with the burst traffic which has been triggered by the sensors. ${ }^{[9]}$. Some of the scientists (2009) have done experiments on human live cells.After the successful theoretical study, they proposed a novel human implantable neural recording system which can extract the power from live human cell and supply the same power for the biomedical neural recording system ${ }^{[10]}$.

\section{Materials and Methods}

In order to design liquid based electronic circuits, synthetic plasma (liquid) has been developed in applied science laboratories. This product has been made as alternative of red blood cells. Here special care has been taken to maintain similar chemical composition. Depending upon the type of synthetic plasma, it can be developed in different methods with the help of chemical isolation, recombinant bio technology or synthetic production. Developed synthetic plasma has similar characteristics as conventional red blood cells so developed liquid can be used for current conduction. The developed synthetic plasma must have the following characteristics. First, it must be reliable for use and human friendly in nature for any kind of blood groups. Second, it also means that plasma can be processed to remove all diseasecausing agents such as viruses and microorganisms. Third, it must be shelf stable which is contrast to natural blood because in natural blood clouting takes place if temperature differs or after a longer time.

Human blood is a very good conductor for electricity. Wegmueller and his team $(2007,2009)$ experienced that human body can be used as a wireless communication medium for short distance communication ${ }^{[11-12]}$. Current coupling is possible in human body parts like muscle and human blood said by Wegmueller (2010) ${ }^{[13]}$.Ethically it is not possible to use actual human blood for experimental purpose so artificial blood like synthetic plasma has been made in applied science laboratories which has similar characteristics like human blood. Different density plasma liquid has been produced and analyzed to check the stability of plasma liquid. The intension behind different density liquid is to make a human friendly circuit which can be implanted in to the human body having different blood groups.

\section{Conventional resistor-transistor logic circuit}

The ideal resistor transistor logic circuit has been shown in Fig 1. It shows two- input gate circuit which can be also called as resistor transistor logic (RTL).RTL circuit is very known technology but to prove that liquid based gate circuit is possible, analysis and designing was carried out from synthetic plasma based resistor transistor logic circuit.

Some scientists $(2005,1998)$ proved that using the ionic property of human body fluids, circuit implantation is possible in to humans ${ }^{[14-15]}$. Kosta 
and his team $(2013,2016)$ successfully noted that desire response of low frequency electronic circuits based on liquid medium can be achieved with proper instrumentation ${ }^{[16-17]}$. Fig 2 depicts the layout of resistor transistor logic circuit using synthetic plasma as liquid medium. Here two big beakers are filled with liquid plasma where three copper wires are inserted in it. Copper wires are shouldered with thin flat rectangular copper strips in order to form terminals of transistor (Emitter, Base and Collector). Similar fashion, other small beakers are filled with same liquid where copper probes are inserted which forms $\mathrm{RB}_{1}, \mathrm{RB}_{2}$ and $\mathrm{R}_{\mathrm{C}}$. To maintain the stability among all liquid components, similar amount of liquid is filled. As shown in figure, wooden stand is used to provide mechanical support to the circuit. It has been noticed that to avoid unwanted ionization in the liquid, all possible experimental steps should be finished within short time after inserting copper wires in to the liquid. All supporting devices like power supply and multimeter are kept very nearer to the physical structure of the circuit to avoid complex connection. Proper instrumentation is required to make these kinds of circuit commercialized. Conventional power supply is provided to the appropriate terminals and results have been analyzed.

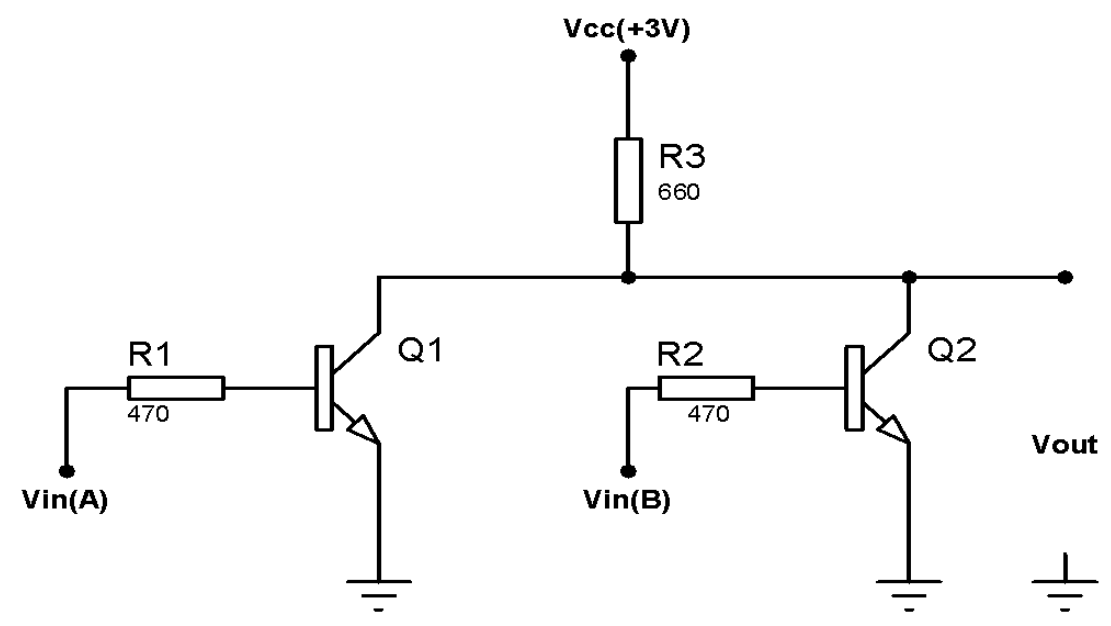

Figure 1: Ideal resistor transistor logic (RTL) circuit

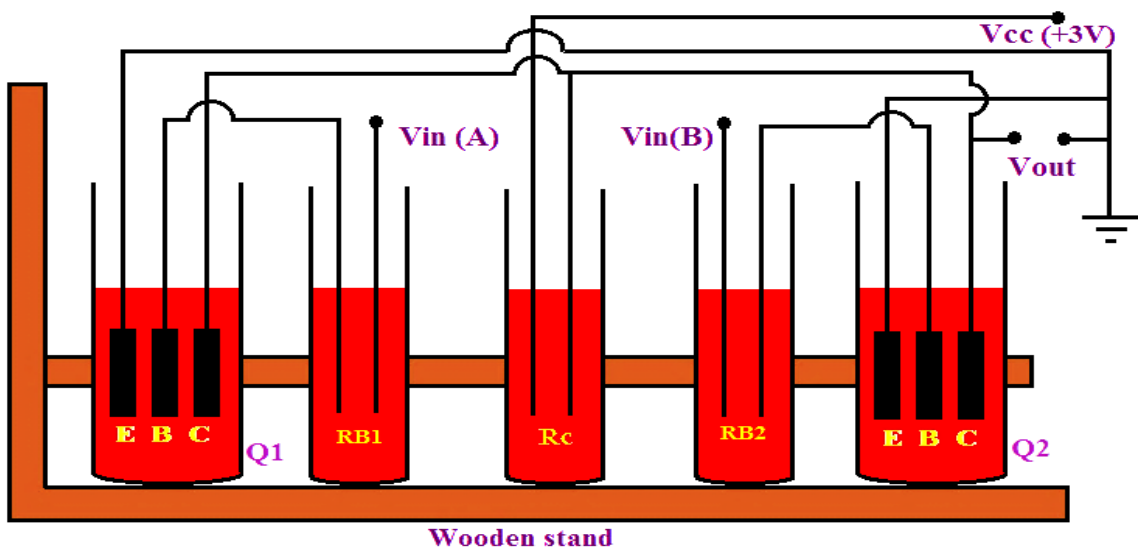

Figure 2: Liquid based resistor transistor logic (RTL) circuit layout 


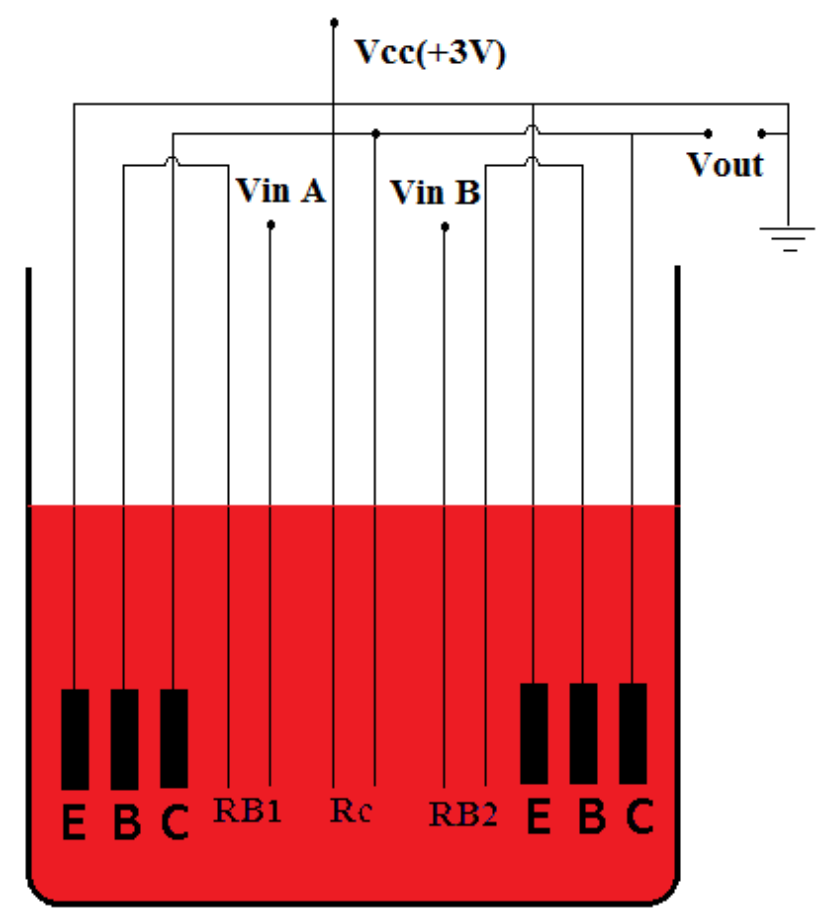

Figure 3: Liquid IC layout for RTL circuit

\section{Concept of liquid Integrated circuit}

After succefull demostration of liquid base electronic circuit,the authors have decided to develop liquid based electronic integrated circuit.The concept of liquid integrated circuit is presented in Fig 3. Instead of inserting copper probe wires in separate beakers, all required probes are inserted in a big size beaker filled with conducting plasma liquid. In presented figure, distance is very important in order to develop individual componenet so proper mechanical instrumentation is required to stabilise all the copper wires in the circuit with ideal location. In order to demostrate the physical model of the liquid integrated circuit, one possible approach is to make a wooden block having holes in it. These wholes should slightly marginally bigger than the thin diameter silicon tube so tubes can be easily pass to those holes of wooden block and would be inserted in to the filled liquid in big beaker. Here of course, wooden block should be kept on the top of the beaker so stability of the physical model can be maintained. Here also suppy voltage has been given from power supply equipment and results have been observed in multimeters.

\section{First step towards pure biological electronic circuit}

From the previous sections, it is evident that liquid base electronic circuit is feasible and it can be successfully demonstrated. One of the applications of synthetic plasma based electronic circuit is human implantation. Since all previous circuits contained copper wires, it cannot be directly implanted in human body. If copper wires would be replaced by any human implantable material then only circuit implantation in to human body is possible. This chapter gives one of the alternatives of this problem. Here in this chapter, copper wires have been replaced by very thin diameter silicon, human implantable tubes which are filled with synthetic plasma. Fig 4 and 5 shows primary experiment where small diameter silicon tubes can be tested as alternatives of copper wires. 


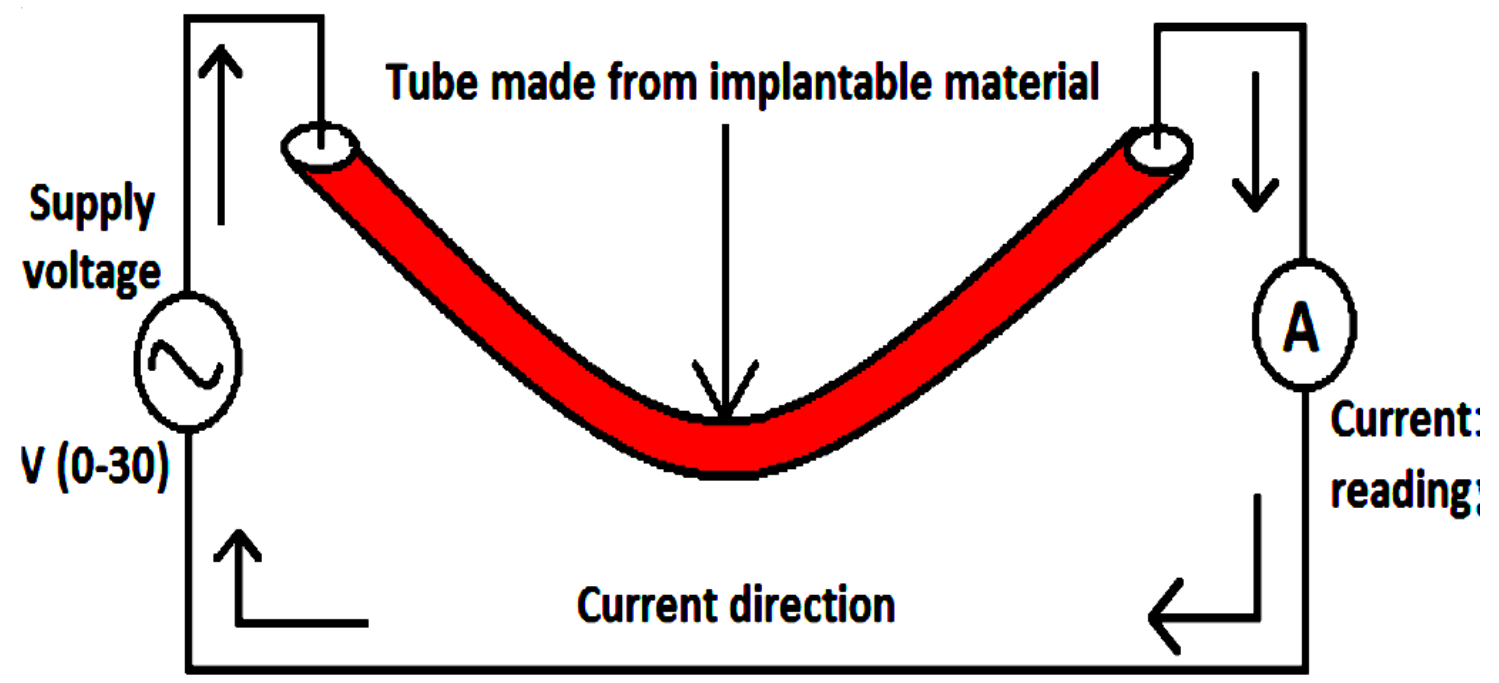

Figure 4: Primary set up for V/I characteristics

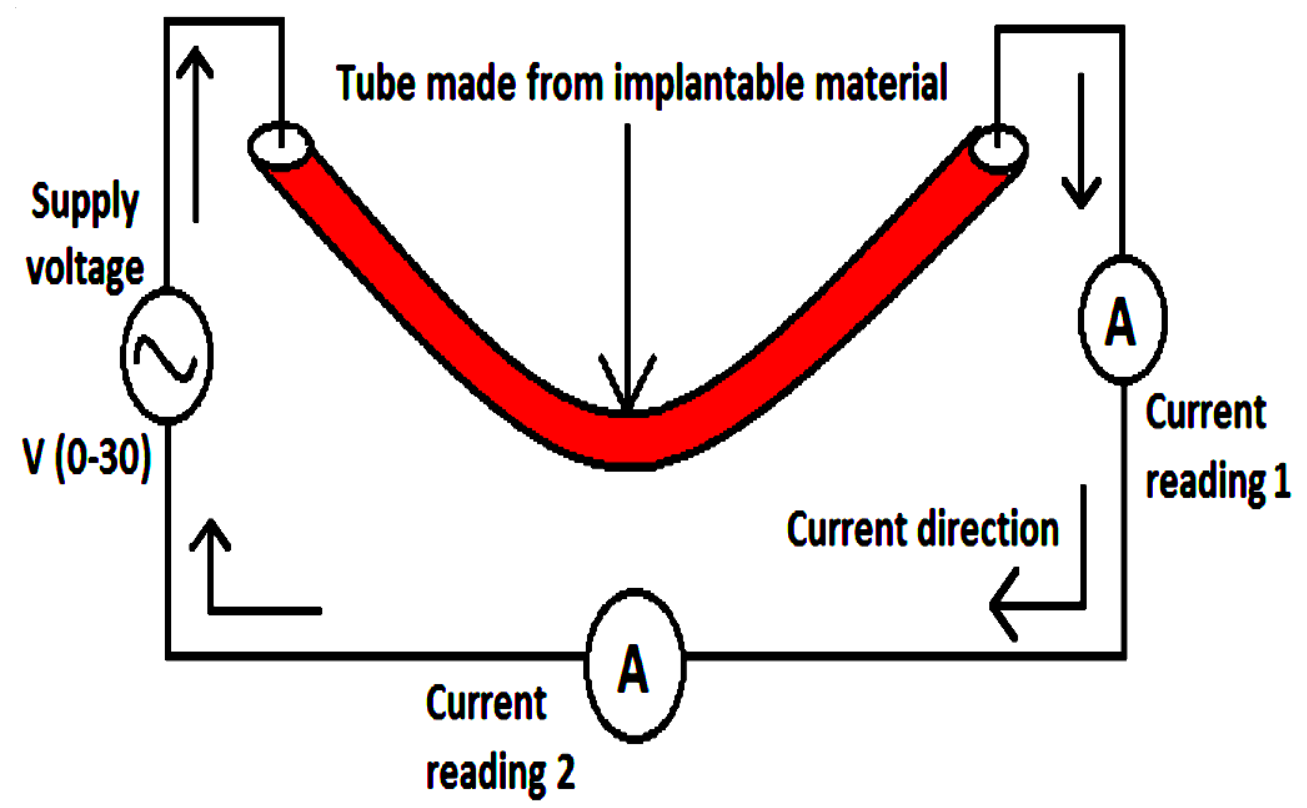

Figure 5: Primary set up for two current readings against voltage

After sufficient searching for copper wires alternative, a very thin diameter ( 2 to $3 \mathrm{~mm}$ ) silicon tubes have been found. It can be utilized for current realization against voltage variation. The Fig 4 shows primary arrangement to get current characteristics for different voltage variation. Fig 4 and 5 depicts that copper wires can be replaced with human implantable material. To determine the resistivity of a thin diameter silicon tubes against the distance, second phase of experiment has been performed and measure result is closely observed.
This experiment lay out is shown in Fig 5. Here both the ammeters are connected in series and $10 \mathrm{~cm}$ distance apart. Here in both the cases, according to the Ohm "es law resistivity increases as the distance increases. The closer look of table tells the whole story. In both the ammeters, current increase with voltage increases but ammeter 2 is connected very far from the positive voltage source so current increment in ammeter 2 is less compared with ammeter 1 . 


\section{Results and discussion}

The table 1 shows the output analysis against the possible input states. It is very clear that possible four inputs can be given to excite the circuit. So here, four combinations (LL, LH, HL and $\mathrm{HH}$ ) is used and output is obtained. Output is considered as high if voltage is above 1.5 Volt and is considered as low if voltage is below 1.5 Volt. So from the table it is very clear that like ideal RTL circuit, output is high only if both the inputs are low otherwise for all other input combination, the output is low. The investigation has been repeated three times with different density liquids for maintaining uniformity and it gives almost similar reaction which supports our novel idea to make pure biological human implantable electronic circuit based on human blood.

From the Fig 4, result has been carried out and presented successfully in table 2 . It depicts very usual linear relation between voltage and current. So conclusion could be made that even copper wires can be replaced by thin diameter silicon tubes filled with conductive liquid. In table 2 voltage is measure in volts and current is measured in $\mathrm{mA}$.

Table 1: Output voltage against two input voltages for different densities

\begin{tabular}{|c|c|c|}
\hline \multicolumn{3}{|c|}{1.2 litre density synthetic plasma } \\
\hline Input voltage 1(volt) & Input voltage 2(volt) & Output voltage(volt) \\
\hline Low & Low & 1.55 (High) \\
\hline Low & High & 0.61 (Low) \\
\hline High & Low & 0.02 (Low) \\
\hline High & High & 0.01 (Low) \\
\hline \multicolumn{3}{|c|}{1.3 litre density synthetic plasma } \\
\hline Input voltage 1(volt) & Input voltage 2(volt) & Output voltage(volt) \\
\hline Low & Low & 1.64 (High) \\
\hline Low & High & 0.65 (Low) \\
\hline High & Low & 0.04 (Low) \\
\hline High & High & 0.03 (Low) \\
\hline & 1.5 litre density synthetic plasma \\
\hline Input voltage 1(volt) & Input voltage 2(volt) & Output voltage(volt) \\
\hline Low & Low & 1.75 (High) \\
\hline Low & High & 0.75 (Low) \\
\hline High & Low & 0.07 (Low) \\
\hline High & High & 0.06 (Low) \\
\hline
\end{tabular}

In presented RTL circuit, lower input will cut off the associated transistor and higher input saturates the associated transistor so if any of the transistor is in saturation region, the output is low. If both the transistors are connected to a low input voltage then directly supply voltage appears as an output. So according to the theory, output is at high level only if both the inputs are at low level. All possible analysis has been done for different densities which are shown in resulting table 1 .

Table 3 shows the desired response when two ammeters are connected in series. Presented work supports the novel idea to demonstrate pure biological electronic circuit which can be implanted in to the human body without any clinical damage. In similar kind of demonstration, step by step approach should have been applied. In such kind of 
experiments, metal should be removed from the biological electronic circuits because metal probably cannot be accepted by human body unless under extremely high clinical care. So in that context firstly, the copper wires can be removed with very thin diameter tubes filled with conducting liquid material. After each and every designing approach, circuit response should be measured so if any practical steps reduce the circuit performance than other alternate can be utilized. After successful replacement of copper wires, thin diameter silicon tubes should be place properly with minimum distance so capacitor and resistor should be manifest. So individual electronic components should be first replaced by the pure biological material and eventually whole circuit can be transformed from metallic structure to human friendly biological circuit.

Table 2 shows current increases linearly as voltage increases. Here synthetic plasma liquid should be carefully inserted inside the very thin diameter tubes by injection and it should be kept within the tube without leakage itself is a challenging issue. Here in both the cases, according to the $\mathrm{Ohm}^{\text {ee }} \mathrm{s}$ law resistivity increases as the distance increases. The closer look of table tells the whole story. In both the ammeters, current increase with voltage increases but ammeter 2 is connected very far from the positive voltage source so current increment in ammeter 2 is less compared with ammeter 1 .

Table 2: V I Characteristics

\begin{tabular}{|c|c|}
\hline Input voltage(v) & Current $(\mathrm{mA})$ \\
\hline 0 & 0 \\
\hline 1 & 0.017 \\
\hline 2 & 0.049 \\
\hline 3 & 0.077 \\
\hline 4 & 0.11 \\
\hline 5 & 0.14 \\
\hline 6 & 0.17 \\
\hline 7 & 0.2 \\
\hline 8 & 0.23 \\
\hline 9 & 0.29 \\
\hline 10 & 0.3 \\
\hline 11 & 0.34 \\
\hline 12 & 0.38 \\
\hline 13 & 0.41 \\
\hline 14 & 0.45 \\
\hline 15 & 0.49 \\
\hline 20 & 0.67 \\
\hline 25 & 0.84 \\
\hline 30 & 1.03 \\
\hline
\end{tabular}


Table 3: V I Characteristics (two currents)

\begin{tabular}{|c|c|c|}
\hline Input Voltage(v) & Current reading $1(\mathrm{~mA})$ & Current reading $2(\mathrm{~mA})$ \\
\hline 0 & 0 & 0 \\
\hline 1 & 0.02 & 0.016 \\
\hline 2 & 0.05 & 0.039 \\
\hline 3 & 0.07 & 0.06 \\
\hline 4 & 0.11 & 0.094 \\
\hline 5 & 0.14 & 0.11 \\
\hline 6 & 0.17 & 0.14 \\
\hline 7 & 0.2 & 0.17 \\
\hline 8 & 0.23 & 0.2 \\
\hline 9 & 0.26 & 0.23 \\
\hline 10 & 0.3 & 0.26 \\
\hline 11 & 0.33 & 0.283 \\
\hline 12 & 0.36 & 0.311 \\
\hline 13 & 0.4 & 0.34 \\
\hline 14 & 0.43 & 0.37 \\
\hline 15 & 0.47 & 0.4 \\
\hline 20 & 0.63 & 0.542 \\
\hline 25 & 0.74 & 0.67 \\
\hline 30 & 0.91 & 0.607 \\
\hline
\end{tabular}

\section{Conclusion}

Emerging technologies and growth of biomedical science can make realization of liquid based electronic circuits. Liquid conductivity can be observed and can be effectively used in order to form different electronic component like $\mathrm{R}, \mathrm{L}$ and C. Human implantable synthetic plasma can be easily made in laboratory. The primary experiment shows that various electronic components can be demonstrated by inserting thin copper wire probes inside the beaker which is filled with synthetic plasma. So eventually, liquid based resistor transistor circuit has been successfully analyzed and presented in this paper. From the results, it is clear that liquid based electronic circuits are feasible with proper mechanical instrumentation. It is also possible to develop compact liquid integrated circuit which gives desire response. Presented liquid IC would be viewed as a sub branch of liquid state electronics. The circuit has been analyzed for different density liquids and it shows similar 
response which supports the novel idea to develop pure biological electronic circuit which can be implanted in to humans with proper clinical care. The proposed work claims about the development and designing of liquid based novel circuit for human machine interface.

Liquid based electronic circuits have scope of research. There is a huge gap between basic electronic circuits and biomedical engineering. Presented research is the link between said fields. Presented research utilised synthetic plasma as a liquid to design and developed electronic components and eventually whole electronic circuits. Presented research has limitation of providing conventional and compact liquid based electronic circuits because human made circuit has been presented and demonstrated here. So in future, proper mechanical instrumentation of similar kind of circuits is required for producing similar kinds of pure biological electronic circuits. Presented biological electronic circuits have great and noble applications in medical society without any clinical damage. Biological electronic circuits can be implanted in to living human body with great medical care in order to replaced damage tissues or body parts.

\section{Acknowledgements}

Authors are extremely thankful to Gujarat Council on Science and Technology (GUJCOST) for providing financial support.

\section{References}

1. Kosta, S; Kosta, Yogesh; Bhatele, Mukta; Dubey, Y; Gaur A; Kosta, S.; Gupta, J; Patel, Amit; and Patel, B.; Human blood liquid memristor.International Journal of Medical Engineering and Informatics 2011,3(1),16-29.

2. Kosta,S; Dubey, A; Gupta, P; Nair, P; Kosta, S; Chaudhary, J; Patel, B.First physical model of human tissue skin based memristors and their network. International Journal of Medical Engineering and Informatics 2013,5(1),5-19.

3. Kosta, S; Bhatele, M; Chuadhari, J; Upadhyaya, T; Soni, D; Kosta, Y.Human blood-based electronic transistor. International Journal of medical engineering and informatics 2012,4(4),373-386.

4. Kosta, S; Kosta, Y; Chaudhary, J; Vaghela, P; Patel, A; Patel, B; Mehta, H.Bio-material human body part (palm fingers) based electronic FET transistor. International journal of biomedical engineering and technology 2012,10(4),368382.

5. Kosta, S. P, Y. P. Kosta, D. Archana, G. Pratik, and P. Piyush. "Human tissue skin based electronic transistor." Int J Biomech Biomed Robot 2012,8-25.

6. Wegmueller MS, Kuhn A, Froehlich J, Oberle M, Felber N, Kuster N, Fichtner W. An attempt to model the human body as a communication channel. IEEE Trans Biomed Eng 2007; 54(10): 1851-1857 [PMID: 17926683 DOI: 10.1109/TBME.2007.893498]

7. Wegmueller M, Felber N, Fichtner W, Lehner A, Hess O, Froehlich J, Kuster N, Reutemann R, Oberle M. Measurement system for the characterization of the human body as a communication channel at low frequency. Conf Proc IEEE Eng Med Biol Soc 2005; 4: 3502 3505 [PMID: 17280979 DOI: 10.1109/IEMBS.2005.1617234]

8. Patil,Y; Pawar, S; Jadhav, S; Kadu, J; Biochemistry of metal absorption in human body: Reference to check impact of nano particles on human being. Int J Sci Res Publ 3 ,2013,1-5.

9. Nie Z, Li Z, Huang R, Liu Y, Li J, Wang L. A statistical frame based TDMA protocol for human body communication. Biomed Eng Online 2015; 14: 65 [PMID: 26155949 PMCID: PMC4495947 DOI: 10.1186/s12938-015-00611]

10. Hmida, G.; Ekuakille, A.; Kachouri, A.; Ghariani, H.;Trotta A.; Extracting electric power from human body for supplying neural recording system. Measurement 4,2009,5-9.

11. Wegmueller, M.; Huclova, S.; Froehlich, J.; Oberle, M.; Felber, N.; Kuster, N.; Fichtner, W.; Galvanic coupling enabling wireless implant communications.IEEE Transactions on Instrumentation and Measurement, 2009,58(8),2618-2625.

12. Wegmueller, M.; Hediger, M.; Kaufmann,T.; Buergin, F.; Fichtner, W.;Wireless implant 
communications for biomedical monitoring sensor network, In Circuits and Systems, 2007. ISCAS 2007. IEEE International Symposium on, IEEE, 2007,809-812.

13. Wegmueller, M.; Oberle, M.; Felber, N.; Kuster, N.; Fichtner, N.;Signal transmission by galvanic coupling through the human body. IEEE Transactions on Instrumentation and Measurement ,2010,59(4),963-969.

14. Mohamad, S.; Hu, Y.;Coulombe, J.;Wireless smart implants dedicated to multichannel monitoring and microstimulation." IEEE Circuits and systems magazine,2005,5(1),2139.

15. Lindsey DP, McKee EL, Hull ML, Howell SM. A new technique for transmission of signals from implantable transducers. IEEE Trans Biomed Eng 1998; 45(5): 614-619 [PMID: 9581060 DOI: 10.1109/10.668752]

16. Kosta SP, Manavadaria M, Pandya K, Kosta YP, Kosta S, Mehta H, Patel J. Human blood plasma-based electronic integrated circuit amplifier configuration. J Biomed Res 2013; 27(6): 520-522 [PMID: 24285951 PMCID: PMC3841478 DOI: 10.7555/JBR.27.20130058]

17. Pandya KV, Kosta S. Synthetic Plasma Liquid Based Electronic Circuits Realization-A Novel Concept. Int J Biomed Sci 2016; 12(3): 79-82 [PMID: 27829822 PMCID: PMC5080411] 